\title{
CONTROLADOR ROBUSTO MULTIOBJETIVO PARA O AMORTECIMENTO DE OSCILAÇÕES ELETROMECÂNICAS EM SISTEMAS ELÉTRICOS DE POTÊNCIA
}

\author{
Ricardo Vasques de Oliveira* \\ vasques@sel.eesc.sc.usp.br
}

\author{
Rodrigo Andrade Ramos*
}

ramosesel.eesc.sc.usp.br

\author{
Newton Geraldo Bretas* \\ ngbretasesel.eesc.sc.usp.br
}

* Departamento de Engenharia Elétrica - Escola de Engenharia de São Carlos - USP

Av. Trabalhador São-carlense, 400 - CEP 13566-590 - São Carlos SP - Brasil

\begin{abstract}
The present paper proposes the application of robust control techniques for the development of a systematic design methodology of multiobjective controllers to damp lowfrequency electromechanical oscillations in electric power systems. The control technique adopted considers the uncertainties regarding to the variations of the electric power system operating conditions. The imposition of an upper bound for the output energy of the closed loop system and the regional pole placement are used as design objectives in the proposed methodology. The upper bound for the output energy is proposed as a performance index in the design of damping controllers for power systems. The design methodology is structured to provide controllers that fulfill various practical requirements of the electromechanical oscillations problem in electric power systems. The presented results demonstrate the effectiveness of the designed controllers.
\end{abstract}

KEYWORDS: Electric power systems, small signal stability, damping controllers, robust control, LMIs.

Artigo submetido em 11/11/2004

1a. Revisão em 25/04/2005;

2a. Revisão em 06/09/2005;

Aceito sob recomendação do Editor Associado

Prof. José Roberto C. Piqueira

\section{RESUMO}

O presente trabalho propõe a aplicação de técnicas de controle robusto para o desenvolvimento de uma metodologia sistemática de projeto de controladores multiobjetivo para o amortecimento de oscilações eletromecânicas em sistemas elétricos de potência. A técnica de controle adotada considera as incertezas referentes às variações das condições de operação do sistema elétrico de potência. A imposição de um limite superior para a energia da saída do sistema em malha fechada e o posicionamento regional de pólos são utilizados como objetivos de projeto na metodologia proposta. O limite superior para a energia da saída é proposto como índice de desempenho no projeto de controlador de amortecimento para sistemas de potência. A metodologia de projeto é estruturada de forma a gerar controladores que atendam a vários requisitos práticos do problema de oscilações eletromecânicas em sistemas elétricos de potência. Os resultados obtidos demonstram a eficiência dos controladores projetados pela metodologia proposta.

PALAVRAS-CHAVE: Sistemas elétricos de potência, estabilidade a pequenas perturbações, estabilizadores de sistemas de potência, controladores de amortecimento, controle robusto, desigualdades matriciais lineares. 


\section{INTRODUÇÃO}

A resposta transitória dos sistemas elétricos de potência, em geral, é caracterizada por oscilações eletromecânicas de baixa freqüência. Estas oscilações são prejudiciais para a operação dos sistemas elétricos de potência, pois as mesmas podem dificultar a operação do sistema em condições transitórias. Tais oscilações podem, em alguns casos, gerar limitações nas transferências de potência entre as barras do sistema. Além disto, as oscilações de baixa freqüência, quando mal amortecidas, podem causar a perda de sincronismo dos geradores. No entanto, os efeitos adversos causados pelas oscilações podem ser reduzidos através do amortecimento das mesmas. Além de estender o limite de transferência de potência, em condições transitórias, o amortecimento das oscilações proporciona uma operação estável e segura dos sistemas elétricos de potência submetidos a pequenas perturbações, permitindo assim o contínuo fornecimento de energia elétrica.

Em (DeMello e Concordia, 1969) foi proposta a utilização de um controle suplementar para fornecer amortecimento adicional às oscilações eletromecânicas do sistema. Tal controlador tem como entrada o desvio de velocidade angular do rotor do gerador (ou outro sinal proporcional à velocidade) e como saída um sinal estabilizante que é adicionado à referência de tensão dos Reguladores Automáticos de Tensão (ou AVRs, do Inglês Automatic Voltage Regulators). O controlador proposto em (DeMello e Concordia, 1969) será chamado, neste trabalho, de Estabilizador Clássico (EC) pelo fato de utilizar uma técnica de controle clássico em seu procedimento de projeto.

Um dos maiores problemas inerentes ao projeto de controladores lineares para sistemas elétricos de potência vem do fato de que o desempenho dos controladores depende significativamente do ponto de operação do sistema, e as condições de operação do sistema variam normalmente ao longo do dia. Esta característica do sistema dificulta a obtenção de um desempenho desejado para os controladores projetados através das técnicas de controle clássico (Rogers, 2000), dado que tais técnicas baseiam-se em uma linearização do modelo do sistema em um ponto nominal de operação, e que esta linearização tem sua validade restrita a uma vizinhança deste ponto.

O problema citado pode ser contornado através do uso de técnicas de controle robusto. Através destas técnicas é possível obter controladores que garantam formalmente a estabilidade do sistema e um desempenho aceitável do controlador frente às variações das condições de operação do sistema dentro de uma região pré-especificada.

Os grandes avanços obtidos nos últimos anos nas áreas de controle, associado à evolução dos microcomputadores, têm possibilitado o desenvolvimento de novas metodologias de projeto de controladores de amortecimento. As novas metodologias baseadas em técnicas de controle robusto possibilitam, em geral, a obtenção de controladores de amortecimento mais eficientes do que os ECs. Técnicas baseadas na síntese $\mu$ (Swarcewicz e Wroblewska-Swarcewicz, 2001), na teoria de controle $\mathrm{H}_{\infty}$ (Ngamroo, 2001), na teoria de controle misto $\mathrm{H}_{2} / \mathrm{H}_{\infty}$ associada à técnica de posicionamento regional de pólos (Werner et al., 2003), na realimentação de saída periódica (Gupta et al., 2003) e nas incertezas paramétricas (Zanchin e Bazanella, 2003), por exemplo, foram utilizadas com sucesso em metodologias de projeto de controladores robustos de amortecimento baseadas em um modelo que consiste de uma máquina síncrona conectada a um barramento infinito (conhecido usualmente como modelo Heffron-Phillips (Heffron e Phillips,1952)).

Também foram propostas novas metodologias de projeto de controladores robustos de amortecimento que consideram as interações das dinâmicas das máquinas do sistema, através do uso do modelo multimáquinas. Em (Rao e Sen, 2000) e (Ramos et al., 2004a), por exemplo, são apresentadas metodologias de projeto de controladores baseadas no posicionamento regional de pólos na forma de desigualdades matriciais lineares (ou LMIs, do Inglês Linear Matrix Inequalities). A síntese $\mu$ (Yue e Schlueter, 2003), a teoria de controle $\mathrm{H}_{\infty}$ (Chuanjiang et al., 2003), e os algoritmos genéticos (Do Bomfim et al., 2000; Abdel-Magid e Abido, 2003) também foram utilizadas em metodologias de projeto baseadas no modelo multimáquinas.

Neste contexto, o presente trabalho propõe a aplicação de uma técnica de controle robusto para o desenvolvimento de uma metodologia sistemática de projeto de controladores multiobjetivo para o amortecimento de oscilações eletromecânicas em sistemas elétricos de potência. O modelo multimáquinas é utilizado na metodologia de projeto proposta com intuito de evitar os problemas de ajuste e coordenação na etapa posterior ao projeto dos controladores. O procedimento de projeto dos controladores é baseado em técnicas lineares de controle, pois tais técnicas apresentam, em geral, uma maior simplicidade tanto na fase de projeto do controlador quanto na fase de implementação do mesmo. A metodologia de projeto é estruturada de forma a gerar controladores que atendam as restrições práticas do problema em questão.

Algumas destas restrições práticas já foram abordadas em (Ramos et al., 2004a). Nesta referência, foram tratados os requisitos de descentralização, realimentação de saída e ganho nulo em regime permanente, e o objetivo de controle foi equacionado na forma de um posicionamento regional de pólos (PRP).

No presente artigo, propõe-se o projeto de controladores ro- 
bustos para o amortecimento de oscilações eletromecânicas considerando a imposição de um limite superior para a energia da saída do sistema como objetivo de projeto. A imposição de um limite superior para a energia da saída reflete-se diretamente na diminuição das oscilações na saída adotada. Portanto, para reduzir os efeitos adversos causados pelas oscilações eletromecânicas basta adotar uma saída do sistema na qual as oscilações exerçam uma influência significativa, e impor um limite superior para a energia desta saída.

A metodologia de projeto é formulada considerando também o objetivo de projeto apresentado em (Ramos et al., 2004a). Ou seja, além do limite superior para a energia da saída como objetivo de projeto, o posicionamento regional de pólos é adotado para que o sistema em malha fechada apresente um fator de amortecimento mínimo. Com isso, reforçam-se os objetivos de projeto no sentido da obtenção de controladores capazes de amortecer rápida e eficientemente as oscilações eletromecânicas nos sistemas de potência. O problema de controle é agora formulado como uma busca sujeita a restrições na forma de LMIs (Boyd et al., 1994), sendo esta busca orientada pelo atendimento simultâneo de dois objetivos de controle.

Este artigo está estruturado da seguinte forma: a seção 2 apresenta o modelo multimáquinas utilizado na metodologia proposta; a seção 3 apresenta as técnicas de controle utilizadas na metodologia proposta; a seção 4 apresenta a metodologia de projeto proposta; a seção 5 apresenta os testes e os resultados obtidos com os controladores projetados; a seção 6 expõe as conclusões e os comentários finais.

\section{MODELO MULTIMÁQUINAS ADOTADO}

Os estudos de estabilidade a pequenas perturbações, envolvendo análise e projeto de controladores, em geral, são realizados através de modelos matemáticos que descrevem o comportamento do sistema elétrico de potência de interesse. Um mesmo sistema pode ser representado por diferentes modelos, dependendo do objetivo do estudo proposto. As metodologias de projeto de controladores de amortecimento existentes utilizam, em geral, dois tipos básicos de modelos de sistemas elétricos de potência: modelo que consiste de uma única máquina conectada a um barramento infinito e modelo multimáquinas.

Quando se utiliza um modelo de uma máquina conectada a um barramento infinito no procedimento de projeto, surgem problemas relacionados com o ajuste e a coordenação dos controladores projetados. O ajuste e a coordenação do controlador, posteriores ao projeto, são necessários pelo fato de tal modelo não considerar as interações dinâmicas das outras máquinas do sistema. Para evitar tais problemas, este trabalho utiliza o modelo multimáquinas diretamente na etapa de

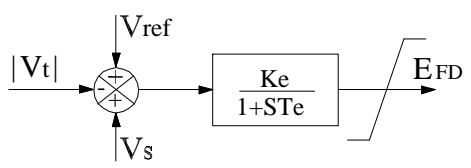

Figura 1: Diagrama de blocos do regulador de tensão utilizado.

projeto do controlador.

Um sistema elétrico de potência multimáquinas é constituído, essencialmente, pelos geradores, linhas de transmissão, cargas e controladores. No modelo multimáquinas adotado, o sistema de transmissão foi modelado como um circuito passivo através de equações algébricas. As cargas do sistema foram modeladas como impedância constante. As máquinas do sistema foram modeladas através do modelo simplificado de um eixo (Padiyar, 1996). Foram também utilizados reguladores automáticos de tensão de primeira ordem. O diagrama de blocos dos reguladores utilizados é apresentado na Figura 1.

Na Figura 1, $|\mathrm{Vt}|$ é o módulo da tensão na barra terminal, Vref é a tensão de referência para o regulador de tensão e Vs é o sinal estabilizante do controlador de amortecimento (entrada de controle do AVR). Nas simulações utilizaram-se limites de $+5,0$ p.u. e $-5,0$ p.u. para as tensões de campo.

As equações algébricas do modelo são eliminadas através de um processo de redução da rede de transmissão. O conjunto de equações que descreve o comportamento do sistema de potência multimáquinas utilizado neste trabalho, após tal redução, é dado por:

$$
\dot{\delta}_{i}=\omega_{0} \omega_{i}-\omega_{0}
$$

$$
\begin{array}{r}
\dot{\omega}_{i}=\frac{1}{2 H_{i}}\left[P_{m i}-D_{i} \Delta \omega_{i}-\sum_{k=1}^{m} E_{q i}^{\prime} E_{q k}^{\prime}\left(G_{i k} \cos \left(\delta_{k}-\delta_{i}\right)\right.\right. \\
\left.\left.-B_{i k} \operatorname{sen}\left(\delta_{k}-\delta_{i}\right)\right)\right]
\end{array}
$$

$$
\begin{gathered}
\dot{E}_{q i}^{\prime}=\frac{1}{\tau_{d o}^{\prime}}\left[E_{F D i}-E_{q i}^{\prime}+\left(x_{d i}-x_{d i}^{\prime}\right) \sum_{k=1}^{m}\left(G_{i k} E_{q k}^{\prime} \operatorname{sen}\left(\delta_{k}-\delta_{i}\right)\right.\right. \\
\left.\left.+B_{i k} E_{q k}^{\prime} \cos \left(\delta_{k}-\delta_{i}\right)\right)\right]
\end{gathered}
$$


onde

$$
\begin{aligned}
& \left|V_{t i}\right|=\left[E_{q i}^{\prime 2}+2 E_{q i}^{\prime} x_{d i}^{\prime} \sum_{k=1}^{m} E_{q k}^{\prime}\left(B_{i k} \cos \left(\delta_{k}-\delta_{i}\right)\right.\right. \\
& +G_{i k} \operatorname{sen}\left(\delta_{k}-\delta_{i}\right)+x_{d i}^{\prime 2} \sum_{k=1}^{m} \sum_{l=1}^{m} E_{q k}^{\prime} E_{q l}^{\prime}\left(G_{i k} G_{i l} \cos \left(\delta_{k}-\delta_{l}\right)\right. \\
& \left.\left.\quad+2 G_{i k} B_{i l} \operatorname{sen}\left(\delta_{k}-\delta_{l}\right)+B_{i k} B_{i l} \cos \left(\delta_{k}-\delta_{l}\right)\right)\right]^{1 / 2},
\end{aligned}
$$

nas quais $\delta_{i}, \omega_{i}, E_{q i}^{\prime}$ e $E_{F D i}$ são, respectivamente, o ângulo do rotor, a velocidade angular do rotor, a tensão transitória de eixo em quadratura e tensão aplicada no circuito de campo. As equações apresentadas referem-se à máquina $i$. Informações detalhadas a respeito das equações do modelo apresentado e dos seus respectivos parâmetros podem ser encontradas em (Padiyar, 1996; Anderson e Fouad, 1994; Kundur, 1994).

O modelo apresentado será linearizado em torno de pontos de operação de interesse para permitir a aplicação de técnicas lineares de controle robusto. No procedimento de projeto o sistema multimáquinas é representado por um conjunto de equações lineares no espaço de estados do tipo:

$$
\begin{gathered}
\dot{x}(t)=A x(t)+B u(t) \\
y(t)=C x(t)
\end{gathered}
$$

sendo $x(t) \in R^{n}$ composto pelos desvios das variáveis de estado do sistema com relação a um ponto de operação nominal, $u(t) \in R^{p}$ é um vetor com as entradas de controle do sistema (que neste trabalho corresponde às entradas $V_{s i}$ dos reguladores de tensão), e $y(t) \in R^{q}$ é o vetor de saídas do sistema (que neste trabalho corresponde às derivadas dos desvios de velocidade dos rotores dos geradores).

\section{TÉCNICAS DE CONTROLE ROBUSTO UTILIZADAS}

A técnica de controle utilizada no projeto de controladores de amortecimento tem uma influência significativa no desempenho dos mesmos. Desta forma, torna se importante a escolha de uma técnica de controle que se enquadre dentro dos objetivos de desempenho requeridos pelo problema de oscilações eletromecânicas de baixa freqüência.

No procedimento proposto neste trabalho, para o projeto do controlador de amortecimento, são utilizados dois objetivos de projeto distintos, associados a uma técnica de modelagem capaz de considerar as incertezas referentes às variações do ponto de operação do sistema. Tais objetivos de projeto referem-se, como mencionado anteriormente, ao posicionamento regional de pólos e à imposição de um limite superior para a energia da saída do sistema em malha fechada (para um determinado conjunto de condições iniciais).
Além disso, a metodologia de projeto foi desenvolvida de forma a gerar controladores robustos, descentralizados e baseados na realimentação dinâmica de saída (três requisitos práticos essenciais para o problema de amortecimento de oscilações eletromecânicas em sistemas de potência). Inicialmente, serão apresentados de forma resumida os tratamentos dados aos requisitos acima citados. Mais detalhes sobre esse tratamento podem ser obtidos em (Ramos et al., 2004a; Ramos et al., 2004b).

Um controlador baseado na realimentação dinâmica de saída pode ser representado pelo conjunto de equações lineares no espaço de estados dado por:

$$
\begin{gathered}
\dot{x}_{C}(t)=A_{C} x_{C}(t)+B_{C} y(t) \\
u(t)=C_{C} x_{C}(t)
\end{gathered}
$$

onde $x_{C}(t) \in R^{n}$ é um vetor com os estados do controlador. A equação (8) descreve o comportamento dinâmico do controlador em função da saída da planta $\left(y(t) \in R^{q}\right)$. A equação (9) produz a entrada de controle para o sistema através da aplicação do ganho $C_{C}$ nos estados do controlador.

O procedimento proposto para o projeto do controlador multiobjetivo utiliza uma técnica de modelagem capaz de considerar as incertezas referentes às variações do ponto de operação do sistema elétrico de potência. A técnica utilizada é denominada modelagem politópica (Boyd et al., 1994), e é bastante adequada para o projeto de controladores de amortecimento para sistemas elétricos de potência (Ramos et al., 2002). A modelagem politópica é constituída por um conjunto de $L$ modelos lineares, estruturados a partir da conexão de modelos do tipo (6)-(7) (obtidos a partir da linearização do modelo multimáquinas (1)-(5) em $L$ diferentes pontos de operação) com o modelo de estados do controlador (equações (8)-(9), cujas matrizes devem ser determinadas pelo procedimento de projeto). O conjunto de modelos lineares é dado por

$$
\dot{\tilde{x}}(t)=\tilde{A}_{i} \tilde{x}(t), \quad \tilde{A}_{i}=\left[\begin{array}{ll}
A_{i} & B C_{C} \\
B_{C} C_{i} & A_{C}
\end{array}\right],
$$

onde $\tilde{A}_{i} \in R^{2 n x 2 n}$, para $i=1, \ldots, L$, são as matrizes de estados do sistema em malha fechada, $\tilde{x}(t) \in R^{2 n}$ é um vetor com os estados do sistema e do controlador, e $A_{C}, B_{C}$ e $C_{C}$ são as variáveis matriciais a serem obtidas pelo procedimento de projeto. Uma vez obtidas tais matrizes, o controlador (8)(9) pode ser passado para a forma de função de transferência (mais adequada para a implementação final).

Utilizando a modelagem politópica na metodologia de projeto dos controladores é possível obter controladores de amortecimento capazes de garantir formalmente a estabilidade do sistema elétrico de potência em uma ampla faixa de pontos de operação (robustez de estabilidade). Algumas definições serão apresentadas para um melhor entendimento da 
modelagem politópica utilizada. Considere o modelo dado por

$$
\dot{\tilde{x}}(t) \in \Omega \tilde{x}(t), \quad \tilde{x}(0)=\tilde{x}_{0},
$$

onde $\Omega$ é um conjunto no espaço das matrizes $2 n x 2 n$ formado pela combinação convexa das matrizes $\tilde{A}_{i}$, e $\tilde{x}(0)$ é a condição inicial do sistema em malha fechada. O conjunto $\Omega$, pode ser escrito na forma

$$
\begin{aligned}
\Omega=C o\left\{\tilde{A}_{1}, \ldots, \tilde{A}_{L}\right\} & := \\
& \left\{\sum_{i=1}^{L} \lambda_{i} \tilde{A}_{i} \mid \lambda_{i} \geq 0, \sum_{i=1}^{L} \lambda_{i}=1\right\} .
\end{aligned}
$$

Através de (12) pode-se observar que o conjunto $\Omega$ é um politopo no espaço das matrizes, e as matrizes $\tilde{A}_{i}$ são os vértices deste politopo. A utilização da modelagem politópica na metodologia de projeto do controlador de amortecimento garante a estabilização do sistema em malha fechada, não somente para os pontos de operação utilizados na construção do sistema politópico, mas também para todos os pontos de operação que podem ser gerados a partir da combinação convexa dos vértices do politopo (Ramos et al., 2002).

O tratamento das restrições de descentralização é feito através da imposição de estruturas bloco-diagonais para as matrizes $A_{C}, B_{C}$ e $C_{C}$, associado a uma reformulação das equações de projeto apresentada em (Ramos et al., 2004a). Com isso, são atendidos, no procedimento de projeto, os requisitos práticos essenciais mencionados anteriormente. Em seguida, apresentam-se as bases matemáticas da formulação dos objetivos de projeto utilizados neste trabalho, a qual permite a obtenção de controladores eficazes no amortecimento de oscilações.

A imposição de um limite superior para energia da saída do sistema em malha fechada constitui o primeiro objetivo de projeto usado na metodologia. A imposição deste limite superior para a energia reflete diretamente na resposta oscilatória da saída adotada. As velocidades angulares dos rotores das máquinas síncronas são adotadas como saída do sistema em malha fechada no procedimento de projeto, pois as oscilações eletromecânicas do sistema se manifestam significativamente nestas velocidades. A imposição de um limite superior para a energia destas saídas do sistema em malha fechada reflete na melhoria da resposta da velocidade das máquinas, uma vez que o comportamento oscilatório das mesmas tende a ser reduzido. Conseqüentemente, isso se traduz em uma contribuição para a redução dos efeitos adversos causados pelas oscilações da referida saída.

Para a imposição desse limite superior para a energia da saída, considere o modelo politópico, em malha fechada, dado por (11) e

$$
z(t)=\tilde{C}_{c l} \tilde{x}(t)=\left[\begin{array}{ll}
C_{\omega} & \mathbf{0}
\end{array}\right]\left[\begin{array}{l}
x(t) \\
x_{C}(t)
\end{array}\right],
$$

onde $z(t)$ é a saída do sistema em malha fechada (sendo que a matriz $C_{\omega}$ seleciona as velocidades dos rotores como saídas desse sistema). A máxima energia da saída do sistema (11)(13) para uma dada condição inicial $\tilde{x}(0)$ é dada por

$$
\begin{array}{r}
\max \left\{\int_{0}^{\infty} z^{T}(t) z(t) d t \mid \dot{\tilde{x}}(t)=\Omega \tilde{x}(t), \quad \tilde{x}(0)=\tilde{x}_{0},\right. \\
\left.z=\tilde{C}_{c l} \tilde{x}(t)\right\} .
\end{array}
$$

Suponha que exista uma função quadrática de Lyapunov, dada por

$$
V(\tilde{x}(t))=\tilde{x}^{T}(t) \tilde{P} \tilde{x}(t), \quad \tilde{P}>0
$$

e que

$$
\dot{V}(\tilde{x}(t))=\tilde{x}^{T}(t)\left(\tilde{A}_{i}^{T} \tilde{P}+\tilde{P} \tilde{A}_{i}\right) \tilde{x}(t)<-z^{T}(t) z(t),
$$

para todo $\tilde{x}(t)$ e $z(t)$ satisfazendo o sistema politópico (11) e (13) para $i=1, \ldots, L$. Integrando ambos os lados da desigualdade (16), para o intervalo de tempo $[0, \tau]$, obtémse

$$
V(\tilde{x}(\tau))-V(\tilde{x}(0))<-\int_{0}^{\tau} z^{T}(t) z(t) d t,
$$

para todo $\tau>0$. Para $\tilde{P}>0$ temos que $V(\tilde{x}(\tau)) \geq 0$ e, portanto, conclui-se que $V(\tilde{x}(0))=\tilde{x}^{T}(0) \tilde{P} \tilde{x}(0)$ é um limite superior da máxima energia da saída $z(t)$ para uma dada condição inicial $\tilde{x}(0)$ (Boyd et al., 1994).

Para atender a um maior número de condições iniciais, o problema será estendido para o caso onde o limite superior para a energia da saída do sistema é obtido não somente para uma condição inicial específica, mas sim para um conjunto de possíveis condições iniciais pertencente ao elipsóide $\varepsilon=\left\{\xi \mid \xi^{T} \tilde{W} \xi \leq 1\right\}$, centrado na origem do espaço de estados linearizado. A matriz $\tilde{W}$ é uma ponderação nos estados do sistema para modelar o elipsóide de forma que um conjunto de condições iniciais esteja contido nele. No procedimento proposto para o projeto do controlador, o limite superior para a máxima energia da saída $z(t)$ para um conjunto de condições iniciais pertencente a $\varepsilon$ será dado por uma constante $\bar{\gamma}_{0}$ que satisfaz $\tilde{W}^{-1 / 2} \tilde{P} \tilde{W}^{-1 / 2}<\bar{\gamma}_{0} I$ (Boyd et al., 1994).

É importante mencionar que a minimização da energia da saída poderia ser utilizada (ao invés de um limite superior para a mesma). No entanto, a utilização de um procedimento 
de otimização para se obter a mínima energia da saída adotada pode produzir controladores impraticáveis, dado o esforço de controle que seria necessário para se obter essa mínima energia.

$\mathrm{O}$ valor de um limite superior que forneça um bom desempenho pode ser escolhido a partir do menor limite superior $\left(\bar{\gamma}_{\text {min }}\right)$. O menor limite superior para um determinado sistema garante a menor energia possível para a saída escolhida, e pode ser obtido através da resolução do problema de controle minimizando o limite superior para a energia de saída. $\mathrm{O}$ valor a ser especificado no projeto é escolhido a partir de $\bar{\gamma}_{\min }$ de forma que não gere excessivo esforço de controle, impedindo assim a obtenção de controladores praticáveis em sistemas reais. Cabe ao projetista verificar se o valor especificado é adequado para a obtenção de controladores praticáveis.

É importante ressaltar que o conceito de energia utilizado neste trabalha não se relaciona com o conceito utilizado para comercialização de energia elétrica. O conceito de energia da saída do sistema (energia da velocidade) utilizado neste trabalho está relacionada diretamente com a energia cinética do sistema multimáquinas. Considere a energia da saída definida por:

$$
\begin{aligned}
E_{\text {sá́da }}=\int_{0}^{\infty} z^{T}(t) z(t) d t & = \\
& \int_{0}^{\infty}\left(\Delta \omega_{1}^{2}+\cdots+\Delta \omega_{n}^{2}\right) d t<\bar{\gamma}_{0},
\end{aligned}
$$

onde $\Delta \omega_{i}$ é o desvio de velocidade da $i$-ésima máquina com relação à velocidade síncrona. A energia cinética do sistema multimáquinas é dada por

$$
E_{\text {cinética }}=\frac{1}{2} J_{1} \omega_{1}^{2}+\cdots+\frac{1}{2} J_{n} \omega_{n}^{2},
$$

onde $J_{i}$ é o momento de inércia da $i$-ésima máquina. Comparando a equação (18) com a equação (19) é possível observar que a energia da saída é proporcional ao valor médio do desvio da energia cinética do sistema em relação à condição na qual as máquinas operam na velocidade síncrona. A energia da saída também pode ser vista como a soma do valor rms do desvio de velocidade (elevado ao quadrado) de todas as máquinas do sistema.

O segundo objetivo de projeto a ser combinado com o primeiro consiste na imposição dos pólos do sistema em malha fechada em uma determinada região do plano complexo. Tal técnica é bastante adequada para o problema em estudo, uma vez que o objetivo primário do controlador de amortecimento é amortecer as oscilações eletromecânicas que surgem no sistema elétrico de potência durante o período transitório (tendo, portanto, uma relação direta com a posição dos pólos do sistema em malha fechada).

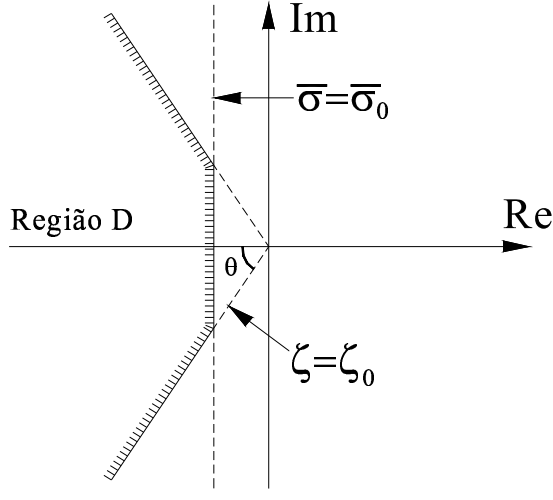

Figura 2: Região para o posicionamento de pólos.

No procedimento de projeto do controlador será utilizada uma região específica (denominada região D), de forma a garantir um fator de amortecimento mínimo e uma taxa de decaimento mínima para os modos de oscilação do sistema em malha fechada. A região D utilizada é definida por $\zeta>$ $\zeta_{0} \mathrm{e} \bar{\sigma}<\bar{\sigma}_{0}$, sendo $\zeta_{0}$ o fator de amortecimento mínimo do sistema politópico em malha fechada, $\bar{\sigma}_{0}$ a taxa de decaimento mínima do sistema politópico em malha fechada. $\mathrm{O}$ fator de amortecimento e a taxa de decaimento dos modos de oscilação estão relacionados diretamente com o decaimento (ou crescimento) da resposta do sistema. Desta forma, o projetista deve escolher estes índices de acordo com o desempenho que o mesmo julgar ser satisfatório para o sistema de potência em estudo. A região $\mathrm{D}$ adotada neste trabalho pode ser visualizada na Figura 2.

Em princípio, o controle poderia ser feito especificando-se como objetivo de projeto apenas a imposição do limite superior para a energia da saída. Isso poderia inclusive levar a resultados menos conservadores, uma vez que apenas um conjunto específico de pólos precisaria ser posicionado para $\mathrm{o}$ atendimento deste objetivo de projeto. No entanto, o fator de amortecimento mínimo é um índice de desempenho usualmente aceito em sistemas de potência como indicativo de uma margem de estabilidade a pequenas perturbações. Por esta razão, o PRP foi combinado à imposição do limite para a energia da saída, nesta proposta de projeto, ficando assim os objetivos da metodologia proposta de acordo com a prática usual em sistemas de potência.

Ambos os objetivos de projeto associados à modelagem modelagem politópica fornecem robustez de desempenho ao sistema em malha fechada. A robustez de desempenho é a capacidade que o sistema em malha fechada tem em manter um desempenho mínimo frente às mudanças no ponto de operação (como, por exemplo, manter um limite superior para a energia da saída e um amortecimento mínimo para os modos de oscilação). 


\section{METODOLOGIA DE PROJETO}

A modelagem politópica, a técnica de posicionamento regional de pólos e a imposição de um limite para a energia da saída do sistema em malha fechada são utilizadas simultaneamente para a formulação de uma metodologia sistemática para o projeto de controladores robustos multiobjetivo para o amortecimento das oscilações eletromecânicas de baixa freqüência.

O problema de controle é formulado como uma busca sujeita a restrições na forma de LMIs. Com o problema na forma de LMIs obtém-se os controladores pela aplicação do solver "feasp", disponível no pacote LMI Lab (Gahinet et al., 1995). Este problema de controle é estruturado a partir da agregação das LMIs correspondentes ao posicionamento regional de pólos e à imposição de um limite para a energia da saída associadas à modelagem politópica. Tal problema de controle consiste basicamente em calcular o controlador dinâmico, representado pelas matrizes $A_{C}, B_{C}$ e $C_{C}$, de forma a garantir os critérios de robustez de estabilidade e robustez de desempenho para o sistema em malha fechada. $\mathrm{O}$ procedimento de projeto é dividido em duas etapas. Inicialmente, determina-se a matriz de ganho de realimentação dos estados do controlador $\left(C_{C}\right)$ e, em seguida, calculam-se as matrizes $A_{C}$ e $B_{C}$, que descrevem a dinâmica do controlador.

O primeiro passo do procedimento de projeto do controlador é a escolha das condições de operação do sistema elétrico de potência que serão utilizadas na construção dos vértices do politopo $\left(\tilde{A}_{i}\right.$, para $\left.i=1, \ldots, \mathrm{L}\right)$. Os critérios de desempenho do controlador $\left(\varsigma_{0}, \bar{\sigma}_{0}\right.$ e $\left.\bar{\gamma}_{0}\right)$ podem ser definidos após a construção dos vértices do politopo. Em seguida, o primeiro conjunto de LMIs (que determina a matriz de ganho de realimentação de estados $C_{C}$ ) pode ser estruturado. $\mathrm{O}$ conjunto de LMIs do problema de controle multiobjetivo referente ao ganho de realimentação de estados $C_{C}$ pode ser resolvido após a construção das variáveis matriciais. O referido conjunto de LMIs é dado por:

$$
\begin{aligned}
& Y_{D}=Y_{D}^{T}>0, \\
& {\left[\begin{array}{cc}
Y_{D} A_{i}^{T}+A_{i} Y_{D}+L_{D}^{T} B^{T}+B L_{D} & Y_{D} C_{\omega}^{T} \\
C_{\omega} Y_{D} & -I
\end{array}\right]<0,} \\
& {\left[\begin{array}{ll}
\bar{\gamma}_{0} I & W^{-1 / 2} \\
W^{-1 / 2} & Y_{D}
\end{array}\right]>0,} \\
& Y_{D} A_{i}^{T}+A_{i} Y_{D}+L_{D}^{T} B^{T}+B L_{D}+2 \bar{\sigma}_{0} Y_{D}<0, \\
& \operatorname{sen} \theta\left(Y_{D} A_{i}^{T}+A_{i} Y_{D} \quad \cos \theta\left(Y_{D} A_{i}^{T}-A_{i} Y_{D}\right.\right. \\
& \left.\left.+L_{D}^{T} B^{T}+B L_{D}\right) \quad+L_{D}^{T} B^{T}-B L_{D}\right) \\
& \cos \theta\left(-Y_{D} A_{i}^{T}+A_{i} Y_{D} \quad \operatorname{sen} \theta\left(Y_{D} A_{i}^{T}+A_{i} Y_{D}\right.\right. \\
& \left.\left.-L_{D}^{T} B^{T}+B L_{D}\right) \quad+L_{D}^{T} B^{T}+B L_{D}\right)
\end{aligned}
$$

para $i=1, \ldots, L$. Os subscritos $D$ nas variáveis matriciais em (20)-(24) indicam que as respectivas matrizes têm estruturas bloco-diagonais de dimensões apropriadas, necessárias para garantir a estrutura descentralizada dos controladores a serem projetados. O conjunto de LMIs (20)-(24) é obtido a partir da aplicação de mudanças de variáveis e do complemento de Schur nas desigualdades matriciais dos problemas de realimentação estática de estados referentes a cada um dos objetivos de projeto adotados (Boyd et al., 1994; Ramos et al., 2004a).

Após a determinação das variáveis $\bar{Y}_{D}$ e $\bar{L}_{D}$ através da resolução das LMIs (20)-(24), a matriz de ganho de realimentação de estados $C_{C}$ pode ser calculada pela equação

$$
C_{C}=\bar{C}_{C}=\bar{L}_{D} \bar{Y}_{D}^{-1}
$$

Em (25), a notação com a barra acima da variável apresentada indica que a mesma já foi obtida, como resultado da aplicação da respectiva etapa de projeto. A mesma notação vale para a seqüência do artigo.

O cálculo da matriz de realimentação de estados $C_{C}$ encerra a primeira etapa do procedimento proposto. Com a matriz de realimentação de estados obtida, inicia-se a etapa correspondente ao cálculo das matrizes que definem a dinâmica do controlador (matrizes $A_{C}$ e $B_{C}$ ). O conjunto de LMIs utilizado para gerar a matrizes $A_{C}$ e $B_{C}$ do controlador, considerando os objetivos de projeto apresentados, é dado por:

$$
\left[\begin{array}{ll}
P_{D} & P_{D} \\
P_{D} & X_{D}
\end{array}\right]>0
$$

$$
\left[\begin{array}{cl}
P_{D} \bar{A}_{k i}+\bar{A}_{k i}^{T} P_{D} & P_{D} A_{i}+\bar{A}_{k i}^{T} X_{D}+C^{T} F_{D}^{T} \\
+C_{\omega}^{T} C_{\omega} & +S_{D}+C_{\omega}^{T} C_{\omega} \\
A_{i}^{T} P_{D}+X_{D} \bar{A}_{k i}+F_{D} C & A_{i}^{T} X_{D}+X_{D} A_{i}+F_{D} C \\
+S_{D}^{T}+C_{\omega}^{T} C_{\omega} & +C^{T} F_{D}^{T}+C_{\omega}^{T} C_{\omega}
\end{array}\right]<0,
$$

$$
\begin{gathered}
\tilde{W}^{-1 / 2}\left[\begin{array}{cc}
X_{D} & P_{D}-X_{D} \\
P_{D}-X_{D} & X_{D}-P_{D}
\end{array}\right] \tilde{W}^{-1 / 2}<\bar{\gamma}_{0} I, \\
{\left[\begin{array}{cc}
P_{D} \bar{A}_{k i}+\bar{A}_{k i}^{T} P_{D} & P_{D} A_{i}+\bar{A}_{k i}^{T} X_{D}+C^{T} F_{D}^{T} \\
+2 \bar{\sigma}_{0} P_{D} & +S_{D}+2 \bar{\sigma}_{0} P_{D} \\
A_{i}^{T} P_{D}+X_{D} \bar{A}_{k i}+F_{D} C & A_{i}^{T} X_{D}+X_{D} A_{i}+F_{D} C \\
+S_{D}^{T}+2 \bar{\sigma}_{0} P_{D} & +C^{T} F_{D}^{T}+2 \bar{\sigma}_{0} X_{D}
\end{array}\right]<0}
\end{gathered}
$$

$$
\left[\begin{array}{llll}
N_{11} & N_{12} & N_{13} & N_{14} \\
N_{12}^{T} & N_{22} & N_{23} & N_{24} \\
N_{13}^{T} & N_{23}^{T} & N_{33} & N_{34} \\
N_{14}^{T} & N_{24}^{T} & N_{34}^{T} & N_{44}
\end{array}\right]<0
$$


sendo que

$$
\begin{gathered}
N_{11}=\operatorname{sen} \theta\left(P_{D} A_{k i}+A_{k i}^{T} P_{D}\right), \\
N_{12}=\operatorname{sen} \theta\left(P_{D} A_{i}+A_{k i}^{T} X_{D}+C^{T} F_{D}^{T}+S_{D}\right), \\
N_{13}=\cos \theta\left(A_{k i}^{T} P_{D}-P_{D} A_{k i}\right), \\
N_{14}=\cos \theta\left(-P_{D} A_{i}+A_{k i}^{T} X_{D}+C^{T} F_{D}^{T}+S_{D}\right), \\
N_{22}=\operatorname{sen} \theta\left(X_{D} A_{i}+A_{i}^{T} X_{D}+F_{D} C+C^{T} F_{D}^{T}\right), \\
N_{23}=N_{14}^{T}, \\
N_{24}=\cos \theta\left(-X_{D} A_{i}+A_{i}^{T} X_{D}-F_{D} C+C^{T} F_{D}^{T}\right), \\
N_{33}=N_{11}, \\
N_{34}=N_{12}, \\
N_{44}=N_{22}
\end{gathered}
$$

$\mathrm{e}$

$$
\bar{A}_{k i}=A_{i}+B \bar{C}_{C} .
$$

As matrizes $A_{C}$ e $B_{C}$ podem ser calculadas depois de encontradas $\bar{P}_{D}, \bar{X}_{D}, \bar{F}_{D}$ e $\bar{S}_{D}$ que satisfaçam (ou seja, que tornem factível) o conjunto de LMIs (26)-(30). As matrizes $A_{C}$ e $B_{C}$ são então dadas por:

$$
\begin{aligned}
& A_{C}=U^{-1} \bar{S}_{D}^{T}, \\
& B_{C}=U^{-1} \bar{F}_{D},
\end{aligned}
$$

onde $U=\bar{P}_{D}-\bar{X}_{D}$. A execução do procedimento descrito nesta seção gera estabilizadores robustos multiobjetivo descentralizados baseados na realimentação dinâmica de saída.

O conjunto de LMIs (26)-(30) é obtido a partir da aplicação de parametrizações e de mudanças de variáveis, apresentadas em (De Oliveira et al., 2000), nas desigualdades matriciais do problema de controle referente a cada um dos objetivos de projeto adotados (Boyd et al., 1994; Ramos et al., 2004a).

É importante destacar que o problema de controle referente ao posicionamento regional de pólos utilizado apresenta uma LMI com dimensões duas vezes maior que as dimensões das LMIs referentes à energia da saída. Esta característica faz com que a solução do problema de controle referente apenas ao limite superior para a energia exija menos dispêndio computacional quando comparado com a solução do problema de controle referente apenas ao posicionamento regional de pólos utilizado.

\section{TESTES E RESULTADOS}

O desempenho dos controladores propostos foi verificado através de testes realizados em dois modelos de sistema de potência bastante conhecidos, para os quais os resultados obtidos através das análises modais e simulações não-lineares

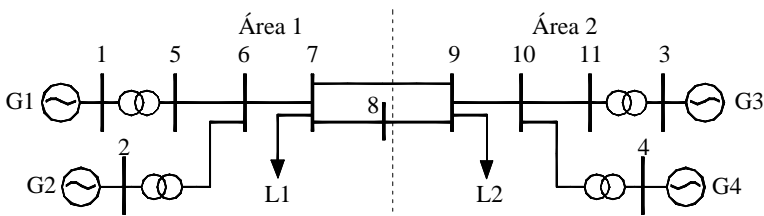

Figura 3: Diagrama unifilar do sistema teste 1.

Tabela 1: Autovalores do sistema em malha aberta.

\begin{tabular}{|c|c|}
\hline Modos de oscilação & Autovalores \\
\hline Inter área & $0.006 \pm \mathrm{j} 2.040$ \\
\hline Local & $0.086 \pm \mathrm{j} 7.407$ \\
\hline Local & $-0.010 \pm \mathrm{j} 6.045$ \\
\hline
\end{tabular}

serão apresentados nesta seção. Ambos os sistemas elétricos de potência adotados são bastante utilizados nos estudos de estabilidade a pequenas perturbações. O primeiro sistema utilizado trata-se de um sistema fictício constituído por duas áreas interligadas através de uma linha de transmissão longa, (conforme mostrado no diagrama unifilar da Figura 3). Os dados completos deste sistema podem ser encontrados em (Kundur, 1994).

O gerador 3 foi utilizado como barramento infinito para fornecer uma referência angular para o sistema. As cargas do sistema nas condições de operação do caso base são $\mathrm{P}_{L 1}=967$ MW, $\mathrm{Q}_{L 1}=100 \mathrm{MVAr} \mathrm{P}_{L 2}=1767 \mathrm{MW}$ e $\mathrm{Q}_{L 2}=100 \mathrm{MVAr}$. Os parâmetros dos reguladores de tensão utilizados no geradores do referido sistema foram $\mathrm{Ke}=200 \mathrm{e} \mathrm{Te}=0.01 \mathrm{~s}$.

Através de análises lineares e não-lineares observou-se que o sistema teste em malha aberta (sem controlador de amortecimento) é instável nas condições de operação do caso base. Desta forma, o sistema requer estabilizadores para operar de maneira estável e com bom desempenho da resposta transitória, quando submetido a pequenas perturbações. Os autovalores referentes aos modos locais e inter área do sistema em malha aberta, nas condições de operação do caso base, são apresentados na Tabela 1 .

Inicialmente foram projetados controladores utilizando apenas o limite superior da velocidade como objetivo de projeto. $\mathrm{O}$ projeto foi realizado de acordo com o procedimento de projeto descrito na seção anterior. No procedimento de projeto dos controladores robustos utilizou-se um politopo com três vértices referentes a três diferentes níveis de carga. Um vértice foi construído utilizando a condição de operação do caso base e os outros dois foram construídos considerando variações de $+10 \%$ e $-10 \%$ nos valores das cargas do caso base. A derivada do desvio de velocidade do rotor dos geradores $\left(\Delta \dot{\omega}_{i}\right)$ foi utilizada como sinal de entrada dos contro- 
Tabela 2: Desvios utilizados para definir o elipsóide.

\begin{tabular}{|c|c|}
\hline Variável de estado & Variação em módulo \\
\hline$\Delta \delta_{i}$ & $0,15 \mathrm{rad}$ \\
\hline$\Delta \omega_{i}$ & $0,05 \mathrm{rad} / \mathrm{s}$ \\
\hline$\Delta E^{\prime} q i$ & 0,15 p.u. \\
\hline$\Delta E_{F D i}$ & 2,00 p.u. \\
\hline
\end{tabular}

ladores robustos para evitar que os mesmos atuem em condições de regime permanente (Ramos et al., 2004b).

O valor para o limite superior da energia foi especificado a partir do menor limite superior calculado para este sistema. O mínimo valor para tal limite é de $0,392\left(\bar{\gamma}_{\min }=0,392\right)$. O critério de desempenho dos controladores propostos foi incorporado no projeto a partir da imposição de um limite superior de $0,9\left(\bar{\gamma}_{0}=0,9\right)$ para a energia das velocidades para um conjunto de condições iniciais contidas no elipsóide $\varepsilon=\left\{\xi \mid \xi^{T} \tilde{W} \xi \leq 1\right\}$. O elipsóide com as condições iniciais foi definido considerando limites para os desvios das variáveis de estado com relação aos valores de tais variáveis em um ponto de equilíbrio do sistema. Os limites adotados para o desvio das variáveis que definem o elipsóide são apresentados na tabela 2.

Para a resolução do conjunto de LMIs utilizou-se o solver "feasp" presente no LMI Control Toolbox do Matlab (Gahinet et al., 1995). O conjunto de LMIs do problema de controle referente ao projeto dos controladores foi resolvido através de um computador com um processador pentium 4 de 1,7 $\mathrm{GHz}$ e o tempo de processamento foi de aproximadamente 9 segundos.

Os pólos referentes aos modos eletromecânicos do sistema equipado com os controladores baseados apenas no limite superior para a energia, na condição de operação do caso base, são apresentados na Figura 4. A resposta não-linear da velocidade angular dos rotores dos geradores do sistema na condição mencionada é mostrada na Figura 5. Para excitar os modos de oscilação, nas simulações não-lineares, foi utilizado um curto-circuito com duração de $32 \mathrm{~ms}$ na barra 8 em $\mathrm{t}=3 \mathrm{~s}$, e em $\mathrm{t}=3,032 \mathrm{~s}$ as linhas 7-8 e 8-9 foram desligas (representando o isolamento do defeito). Decorridos $200 \mathrm{~ms}$ (em t=3,232 s) do isolamento do defeito, o curto-circuito é eliminado e a topologia original da rede é reconstituída através do religamento das linhas.

Os resultados mostraram, conforme o esperado, que apenas o uso do objetivo de projeto referente à energia da saída é suficiente para garantir um desempenho bastante satisfatório. O desempenho dos controladores propostos também foi verificado para os pontos de operação utilizados na construção do politopo (caso base e variações de $\pm 10 \%$ nas cargas da

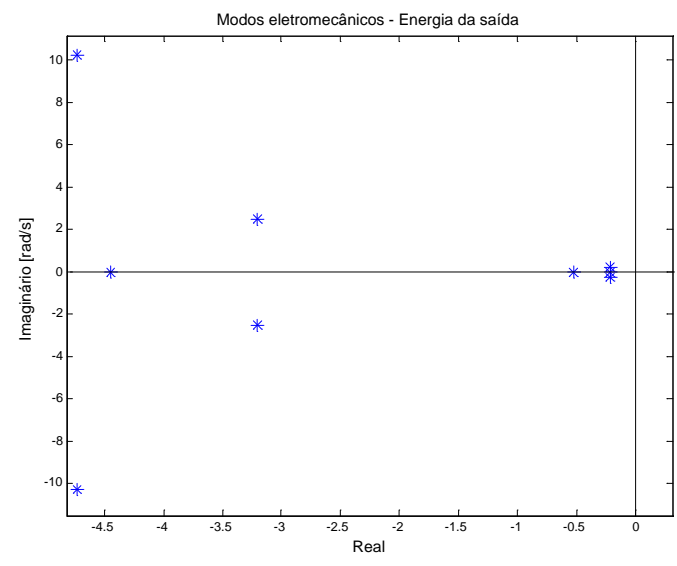

Figura 4: Pólos do sistema equipado com controladores baseados apenas no limite para a energia da saída.

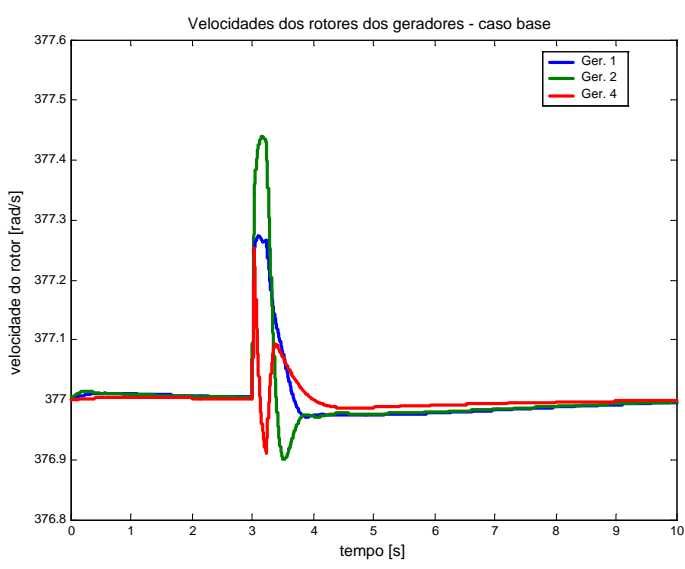

Figura 5: Sistema teste 1 operando com controladores baseados apenas no limite para a energia da saída.

condição de operação do caso base) e para pontos de operação intermediários, onde foram utilizadas variações de $\pm 2 \%$, $\pm 5 \%$ e $\pm 7 \%$ nas cargas da condição de operação do caso base. Os controladores apresentaram um bom desempenho em todas as condições mencionadas.

$\mathrm{Na}$ seqüência, foram projetados controladores incluindo o objetivo de projeto referente ao posicionamento regional de pólos. Desta forma, os controladores projetados atendem a dois objetivos de projeto. O limite superior para a energia da saída utilizado neste caso é igual ao utilizado no projeto anterior, e para o posicionamento de pólos especificou-se um fator de amortecimento mínimo de $5 \%\left(\zeta_{0}=0,05\right)$ e uma taxa de decaimento mínima de $0,01\left(\bar{\sigma}_{0}=0,01\right)$. Os pólos referentes aos modos eletromecânicos do sistema equipado com os controladores multiobjetivo, na condição de operação do 


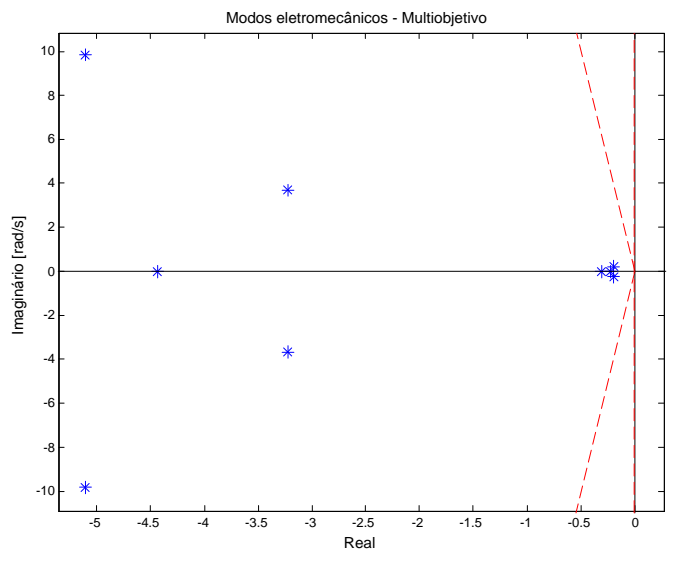

Figura 6: Pólos do sistema equipado com controladores multiobjetivo.

caso base, são apresentados na Figura 6. A resposta nãolinear da velocidade angular dos rotores dos geradores do sistema na condição mencionada é mostrada na Figura 7.

A inclusão do objetivo de projeto referente ao posicionamento regional de pólos não proporcionou melhora significativa no desempeno dos controladores. No entanto, tal objetivo foi incluído pelo fato do fator de amortecimento mínimo ser um índice de desempenho usualmente aceito em sistemas de potência como indicativo de uma margem de estabilidade a pequenas perturbações. Neste caso, o conjunto de LMIs do problema foi resolvido em aproximadamente 1 minuto e 57 segundos.

Foram projetados Estabilizadores Clássicos para os geradores 1,2 e 4 do sistema com o objetivo de comparar o desempenho de tais controladores com desempenho dos controladores propostos. Os ECs foram projetados utilizando-se o modelo Heffron-Phillips e a técnica de sintonia apresentada em (Larsen e Swann, 1981) foi aplicada posteriormente ao projeto dos mesmos. A resposta não-linear da velocidade angular dos rotores dos geradores do sistema equipado com ECs, na condição de operação do caso base, é mostrada na Figura 8.

Como esperado, os ECs foram suficientes para fornecer um bom amortecimento aos modos de oscilação, mesmo em uma condição operativa diferente daquela referente ao ponto de operação nominal de projeto destes estabilizadores. Nota-se, no entanto, que os ECs apresentaram um desempenho inferior ao desempenhos dos controladores propostos, nas condições de operação mostradas. Este fato se repetiu em todas as demais condições operativas testadas.

As funções de transferência dos controladores multiobjetivo

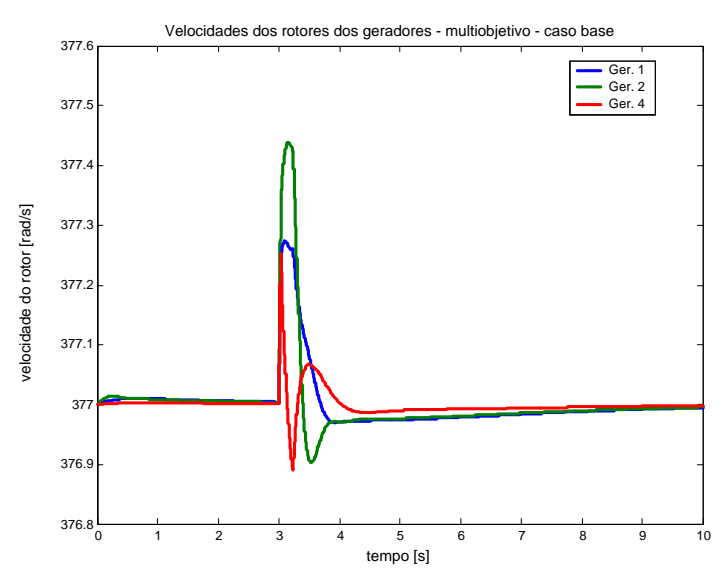

Figura 7: Sistema teste 1 operando com controladores multiobjetivo.

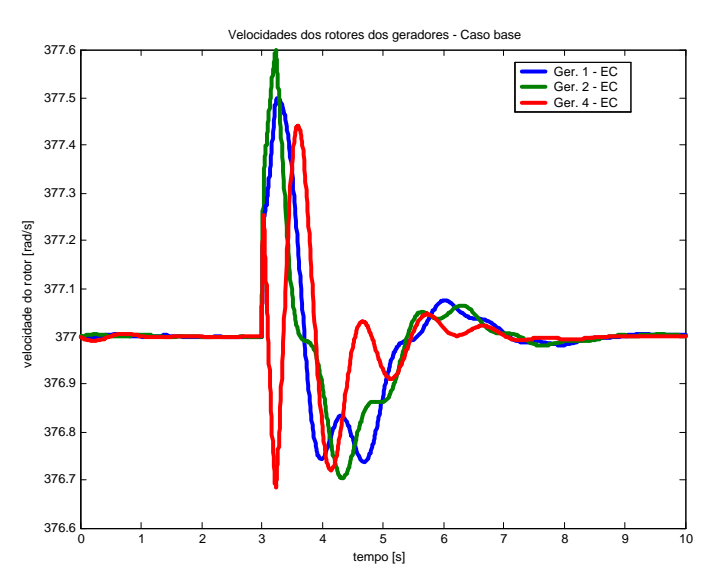

Figura 8: Sistema teste 1 operando com ECs.

propostos e dos ECs projetados para o sistema teste 1 são apresentadas no apêndice.

O segundo sistema utilizado para a realização dos testes trata-se de um sistema constituido por 39 barras e 10 geradores que representa parte do sistema New England / New York. A Figura 9 apresenta o diagrama unifilar do referido sistema, e seus dados completos podem ser encontrados em (Rogers, 2000).

O gerador 10, apresentado na Figura 9, é um equivalente do sistema New York e, por esta razão, tal gerador é utilizado como barramento infinito para fornecer uma referência angular ao sistema. Os parâmetros dos reguladores de tensão utilizados nos geradores do sistema teste 2 foram $\mathrm{Ke}=100 \mathrm{e}$ $\mathrm{Te}=0.01 \mathrm{~s}$. Através de análises modais obsevou-se que o sistema apresenta 9 modos de oscilações, sendo que 8 destes 


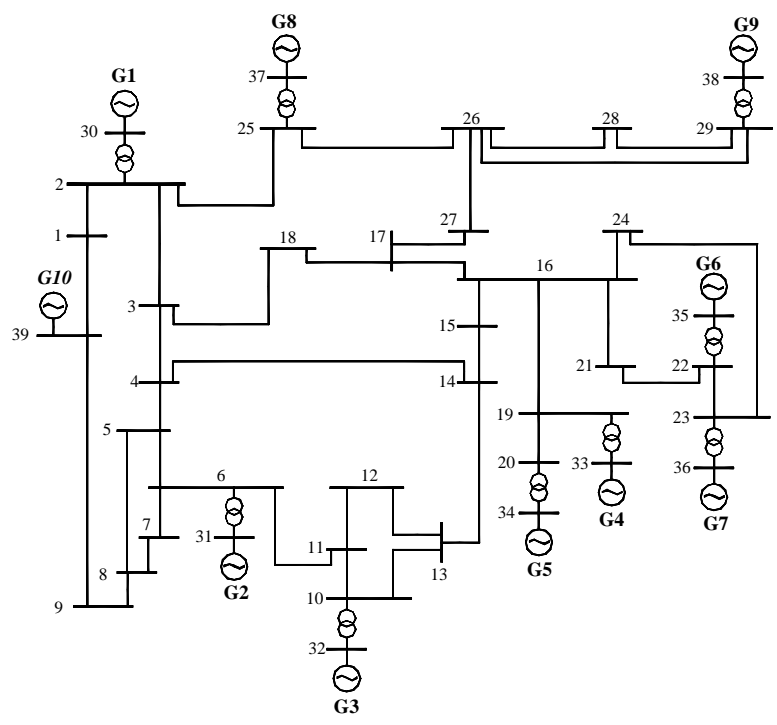

Figura 9: Diagrama unifilar do sistema teste 2.

modos estão na faixa de frequência dos modos locais e 1 está na faixa de frequência dos modos inter área. Todos os modos de oscilação do sistema em malha aberta, nas condições de operação do caso base, são instáveis.

A construção do modelo politópico para o sistema teste 2 foi feita de maneira análoga àquela realizada para o sistema teste 1. Utilizou-se um politopo com três vértices (sendo um caso base e outros dois pontos relativos a variações de $+10 \%$ e $-10 \%$ nos valores das cargas deste caso base), um fator de amortecimento mínimo de 5\% $\left(\zeta_{0}=0,05\right)$, uma taxa de decaimento mínima de $\bar{\sigma}_{0}=0.01$. O mínimo valor do limite superior para a energia da saída, calculado através da minimização de $\bar{\gamma}$, é de $0,489\left(\bar{\gamma}_{\min }=0,489\right)$. No projeto foi especificado um limite superior de $\bar{\gamma}_{0}=1,0$. Neste caso, o conjunto de LMIs do problema de controle referente ao projeto dos controladores foi resolvido em aproximadamente 41 minutos e 20 segundos, no mesmo computador utilizado no projeto para o sistema teste 1 .

A resposta da velocidade angular dos geradores do sistema teste 2 equipado com controladores multiobjetivo (obtida através de simulação não-linear), na condição de operação correspondente ao caso base, é apresentada na Figura 10. Os controladores projetados para o sistema teste 2 também apresentaram um bom desempenho, conforme o esperado.

\section{CONCLUSÕES}

Uma metodologia sistemática de projeto de controladores robustos multiobjetivo para o amortecimento de oscilações eletromecânicas em sistemas elétricos de potência foi apresen-

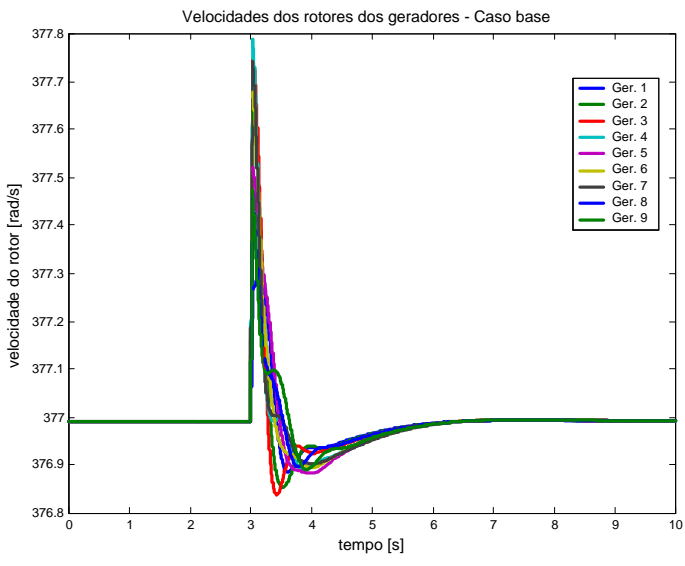

Figura 10: Sistema teste 2 operando com controladores multiobjetivo.

tada. O trabalho propõe, como inovação, um novo índice de desempenho para o projeto de controladores de amortecimento.

Tal metodologia atende a restrições práticas do problema de oscilações eletromecânicas. Além disso, esta metodologia gera controladores que garantem formalmente a estabilidade do sistema e um bom desempenho do controlador frente às variações das condições de operação do sistema. Os controladores obtidos são ainda naturalmente coordenados, devido a utilização do modelo multimáquinas diretamente na etapa de projeto.

O limite superior para a energia do desvio da velocidade dos geradores é proposto como um novo índice de desempenho para o problema de oscilações eletromecânicas em sistemas de potência. Os objetivos incluídos no procedimento de projeto se mostraram adequados ao problema de oscilações eletromecânicas. A imposição de um limite superior para a energia reflete-se diretamente na redução das oscilações da velocidade dos rotores dos geradores do sistema em malha fechada, e conseqüentemente reduz os efeitos adversos causados por tais oscilações. A imposição deste limite, no procedimento de projeto do controlador, garante formalmente a diminuição das oscilações na velocidade dos geradores do sistema para um conjunto pré-determinado de condições iniciais. A solução do problema de controle referente apenas ao limite superior para a energia exige menos dispêndio computacional quando comparando com a solução do problema de controle referente apenas ao posicionamento regional de pólos utilizado. Este menor dispêndio computacional pode se refletir significativamente no tempo computacional requerido para o projeto de controladores envolvendo sistemas de grande porte. 
Através dos resultados e das análises realizadas observouse claramente que os controladores robustos multiobjetivo propostos apresentaram um desempenho bastante satisfatório. Na seqüência deste trabalho, pretende-se tratar aspectos relativos à construção da modelagem politópica e estender a metodologia proposta ao projeto de controladores para dispositivos FACTS.

\section{AGRADECIMENTOS}

Os autores agradecem à FAPESP pelo apoio financeiro concedido a esta pesquisa.

\section{REFERÊNCIAS}

Abdel-Magid, Y. L. e Abido, M. A. (2003). Optimal multiobjective design of robust power system stabilizers using genetic algorithms. IEEE Transactions on Power Systems, v.18, n.3, pp. 1125-1132.

Anderson, P. M e Fouad, A. A. (1994). Power System Control and Stability. IEEE Press.

do Bomfim, A. L. B.; Taranto, G. N. e Falcão, D. M. (2000). Simultaneous tuning of power system damping controllers using genetic algorithms. IEEE Transactions on Power Systems, v. 15, n.1, pp. 163-169.

Boyd, S; El Gahoui, L; Feron, E. e Balakrishnan, V. (1994). Linear Matrix Inequalities in System and Control Theory, SIAM, Philadelphia.

Chiali, M; Gahinet, P. e Apkarian, P. (1999). Robust Pole Placement in LMI Regions. IEEE Transactions on Automatic Control, v.44, n.12, pp. 2257-2270.

Chuanjiang, Z; Khammash, M; Vittal, V. e Wenzheng, Q. (2003). Robust power system stabilizer design using $\mathrm{H} \infty$ loop shaping approach. IEEE Transactions on Power Systems, v.18, n.2, pp. 810-818.

DeMello, F. P. e Concordia, C. (1969). Concepts of synchronous machine stability as affected by excitation control. IEEE Transactions on Power Apparatus and Systems, vol. PAS-88, pp. 316-329.

Gahinet, P; Nemirovski, A; Laub, A. J. e Chiali, M. (1995). LMI Control Toolbox User's Guide. The MATHWORKS Inc..

Gupta, R; Bandyopadhyay, B. e Kulkarni, A. M. (2003). Design of Power System Stabilizer for Single-Machine System Using Robust Periodic Output Feedback Controller. IEE Proceedings - Generation, Transmission and Distribution, v.150, n.2, pp. 211-216.
Heffron, W.G. e Phillips, R.A. (1952). Effect of a Modern Amplidyne Voltage Regulator on Underexcited Operation of Large Turbine Generators. AIEE Transactions on Power Apparatus and Systems, vol. 71, pp. 692-697.

Kundur, P. (1994). Power System Stability and Control. EPRI Editors, McGraw-Hill, New York.

Larsen, E. V. e Swann, D. A. (1981). Applying Power System Stabilizers, parts i, ii and iii. IEEE Transactions on Power Apparatus and Systems, v.PAS-100, n.6, pp. 3017-3048.

Ngamroo, I. (2001). Design of Robust H $\infty$ PSS via Normalized Coprime Factorization Approach. IEEE International Symposium on Circuits and Systems - ISCAS, v.3, pp. 129-132.

de Oliveira, M. C; Geromel, J. C. e Bernussou, J. (2000). Design of Dynamic Output Feedback Decentralized Controllers via a Separation Procedure. International Journal of Control, v.73, n.5, pp. 371-381.

Padiyar, K. R. (1996). Power system dynamics: stability and control. New York : John Wiley \& Sons.

Ramos, R. A; Alberto, L. F. C. e Bretas, N. G. (2004a). A New Methodology for the Coordinated Design of Robust Decentralized Power System Damping Controllers. IEEE Transactions on Power Systems, v.19, n.1, pp. 444-454.

Ramos, R. A; Alberto L. F. C. e Bretas, N. G. (2004b). Decentralized Output Feedback Controller Design for the Damping of Electromechanical Oscillations. Int. J. Electrical Power \& Energy Syst., v.26, n.3, pp. 207219.

Ramos, R. A; Alberto L. F. C. e Bretas, N. G. (2002). Damping Controller Design for Power Systems with Polytopic Representation for Operating Conditions. Proceedings of the IEEE Power Engineering Society Winter Meeting - New York City, USA.

Rao, P. S. e Sen, I. (2000). Robust Pole Placement Stabilizer Design using Linear Matrix Inequalities. IEEE Transactions on Power Systems, v.15, n.1, pp. 313-319.

Rogers, G. (2000). Power System Oscillations. Norwell, MA: Kluwer.

Swarcewicz, A. e Wroblewska-Swarcewicz, K. (2001). Robust Power System Stabilizer. IEEE Power Tech Proceedings, v.2, Porto, Portugal.

Werner, H; Korba, P. e Yang, T. C. (2003). Robust Tuning of Power System Stabilizer Using LMI-Techniques. IEEE Transactions on Control Systems Technology, v.11, n.1, pp. 147-152. 
Yue, M. e Schlueter, R. A. (2003). $\mu$-Synthesis Power System Stabilizer Design Using a Bifurcation Subsystem Based Methodololy. IEEE Transactions on Power Systems, v.18, n.4, pp. 1497-1506.

Zanchin, V. T. e Bazanella, A. S. (2003). Robust output feedback design with application to power systems. Proceedings of the 42nd IEEE Conference on Decision and Control, v.4, pp. 3870 - 3875. Maui, Hawaii USA.

\section{APÊNDICE}

\section{Funções de Transferência}

As funções de transferência dos controladores multiobjetivo projetados para o sistema teste 1 serão apresentadas na forma zero/pólo/ganho, e são dadas por:

$$
\begin{aligned}
& F G 1(s)=22134 \frac{(s+51,91 \pm j 44,21)(s+27,15)(s)}{(s+552,37 \pm j 398,71)(s+54,81)(s+0,20)}, \\
& F G 2(s)=1658,5 \frac{(s+82,60 \pm j 178,90)(s+29,44)(s)}{(s+565,30 \pm j 357,00)(s+72,66)(s+0,23)}, \\
& F G 4(s)=6391,7 \frac{(s+85,16)(s+51,22)(s+9,12)(s)}{(s+62,20 \pm j 49,11)(s+575,97)(s+0,30)} .
\end{aligned}
$$

A função de transferência dos ECs utilizados nos geradores 1,2 e 4 é dada por

$$
F G 124(s)=55,6 \frac{(s+20)(s+0,28)(s)}{(s+66,6)(s+0,24)(s+0,1)} .
$$

